\title{
Etude de la synthèse de films produits par irradiation laser-UV directe
}

\author{
A.L. Thomann, C. Boulmer-Leborgne, J. Hermann, C. Andreazza-Vignolle*, P. Andreazza*, \\ G. Blondiaux** et F. Degas** \\ Laboratoire GREMI, URA 831 du CNRS, Université d'Orléans, BP. 6759, 45067 Orléans \\ cedex 2, France \\ * Laboratoire CRMD, CNRS, rue de Chartres, BP. 6759, 45067 Orléans cedex 2, France \\ ** Laboratoire CERI, CNRS, $3 A$ avenue de la Férollerie, 4507 I Orléans cedex 2, France
}

\begin{abstract}
We have studied the nitriding of titanium by direct laser irradiation, in a nitrogen atmosphere. Two kinds of laser were used : a TEA-CO $\mathrm{CO}_{2}$ emitting in IR at $10.6 \mu \mathrm{m}$ and a XeCl excimer emitting in UV at $308 \mathrm{~nm}$. The power density was in the range $20-80 \mathrm{MW} / \mathrm{cm}^{2}$. In order to relate the caracteristics of the plasma created on the target with the film properties, we have performed surface analyses on the samples. The presence of titanium nitride, oxynitride ( $\mathrm{TiNxOy}$ ) and oxyde $\left(\mathrm{TiO}_{2}\right)$ is evidenced. The use of the $\mathrm{XeCl}$ laser seems to promote the nitriding process against oxydation.
\end{abstract}

Nous avons étudié la synthèse de nitrure de titane par irradiation directe d'une surface de titane, en présence d'azote, avec deux types de laser : un laser $\mathrm{CO}_{2}(\lambda=10.6 \mu \mathrm{m})$ et un laser excimère $\mathrm{XeCl}(\lambda=308 \mathrm{~nm})$. Les intensités laser utilisées vont de 20 à $80 \mathrm{MW} / \mathrm{cm}^{2}$. Lors de l'interaction laser-matière un plasma est initié au dessus de l'échantillon qui interagit avec la surface. Pour comprendre les processus physico-chimiques menant à la synthèse, il est nécessaire de corréler les caractéristiques du plasma avec les propriétés des films synthétisés. Ce plasma, qui est de nature différente selon le laser utilisé, est étudié dans l'article "Direct synthesis of titanium nitride on $\mathrm{T} j$ target surface by laserplasma"; nous nous intéressons ici à la caractérisation des films par différentes techniques d'analyse.

Les états de surface des films synthétisés sont tout d'abord caractérisés par leur rugosité. Elle augmente avec le nombre de tirs et l'intensité laser et est de l'ordre du dixième de $\mu \mathrm{m}$ ou du $\mu \mathrm{m}$. A intensité équivalente et même nombre de tirs, la rugosité est inférieure pour un échantillon synthétisé en UV.

Sur des clichés de microscopie électronique à balayage (échelle de qq $\mu \mathrm{m}$ ) on observe sur la surface traitée des pores et des fissures dont le nombre augmente avec le nombre de tirs et l'intensité. Dans des conditions extrêmes (intensité élevée, grand nombre de tirs), une structure complexe apparaît, composée diîlots séparés par de profondes crevasses, ceci est vrai quel que soit le laser utilisé. 
La microstructure des surfaces (échelle de $\approx 100 \mathrm{~nm}$ ) est obtenue par microscopie en transmission. Les clichés présentent des aspects très différents : soit une juxtaposition de grains octogonaux dont le diamètre augmente de 50 à $150 \mathrm{~nm}$ avec le nombre de tirs et l'intensité, cette structure est majoritaire dans les échantillons synthétisés avec le laser $\mathrm{CO}_{2}$; soit une succession de lamelles $(50 \times 200 \mathrm{~nm})$ contenues dans de gros grains, qui apparaît surtout lorsqu'on utilise le laser $\mathrm{XeCl}$.

Les propriétés cristallographiques sont ensuite mises en évidence par deux techniques complémentaires.

La diffraction des rayons X rasants (GIXRD) permet une détermination globale des phases cristallines sur toute la surface traitée et à différentes profondeurs selon l'angle d'incidence du faisceau X (de la dizaine de $\mathrm{nm}$ à $q q \mu \mathrm{m}$ ). Les phases mises en évidence sont les mêmes quel que soit le laser utilisé, elles correspondent à du titane pur $(\alpha-T i)$, du titane contenant de l'azote en insertion ( $\alpha$-TiN) et du nitrure de titane ( $\delta$-TiN). Dans les échantillons synthétisés avec le laser $\mathrm{XeCl}$, le titane est détecté à la surface alors que dans ceux obtenus avec le laser $\mathrm{CO}_{2}$, il n'apparaît qu'en profondeur, sous le nitrure.

La diffraction électronique, permet une étude plus locale des phases cristallines, sur une surface $\approx 1000 \mathrm{~nm}^{2}$. En plus des phases déjà détectées en GIXRD, cette technique a permis de mettre en évidence la phase $\varepsilon-\mathrm{Ti}_{2} \mathrm{~N}$ notamment dans les structures en lamelles qui seraient une succession : $\varepsilon-\mathrm{Ti}_{2} \mathrm{~N} / \alpha-\mathrm{TiN}$. Les grains octogonaux seraient constitués de $\delta$-TiN.

La composition chimique est déterminée par spectroscopie de photo-électrons $X$ (ESCA) sur une profondeur $\approx 100 \AA$ et, de façon globale sur toute l'épaisseur de l'échantillon, par rétrodiffusion de particules chargées (RBS).

L'ESCA donne des renseignements sur les liaisons chimiques qui existent entre les éléments et permet en particulier de mettre en évidence Ti-N. Elle existe dans deux espèces différentes : le nitrure de titane $\mathrm{TiNx}$ (sans doute sous stoechiométrique en azote) et un oxynitrure du type TiNxOy. L'oxyde $\mathrm{TiO}_{2}$, présent sur la surface de titane avant le traitement, existe également dans les films. La quantité de ces deux espèces oxydées augmentent avec le nombre de tirs et l'intensité laser. On remarque que dans les échantillons soumis au laser $\mathrm{XeCl}$ le nitrure est en quantité un peu plus élevée et plus riche en azote.

En RBS on obtient un dosage de l'azote et de l'oxygène dans tout l'échantillon ainsi qu'une estimation de la profondeur sur laquelle ils sont présents. La concentration de ces éléments est maximale à la surface puis diminue de façon exponentielle avec la profondeur, la quantité totale d'azote augmente avec le nombre de tirs. Selon le laser utilisé des différences importantes apparaissent entre les échantillons : l'épaisseur du film synthétisé, définie comme la profondeur jusqu'à laquelle l'azote est détecté, atteint quelques $\mu \mathrm{m}$ avec le laser $\mathrm{CO}_{2}$ et ne dépasse pas 1 à $2 \mu \mathrm{m}$ avec le laser XeCl. De plus, la quantité d'oxygène présente dans le premier cas est élevée, alors qu'en UV elle est négligeable par rapport à la teneur en azote.

La comparaison que l'on a faite entre les échantillons obtenus par traitement laser $\mathrm{CO}_{2}$ ou $\mathrm{XeCl}$, montre que la lumière UV semble plus efficace pour la nitruration au détriment de l'oxydation. L'épaisseur de la couche obtenue dans ce cas est nettement suffisante pour l'application en dureté de ces films et la structure en lamelles majoritaire en UV possède de bonnes caractéristiques mécaniques [1].

[1] J.E.Sundgren, Thin Solid Films 128 (1985) 21-44. 